\title{
Generating unstable resonances for extraction schemes based on transverse splitting
}

\author{
M. Giovannozzi \\ CERN, CH 1211 Geneva 23, Switzerland \\ D. Quatraro* \\ CERN, CH 1211 Geneva 23, Switzerland, \\ and Dipartimento di Fisica, Università degli Studi di Bologna, V. Irnerio 46, Bologna, Italy \\ G. Turchetti \\ Dipartimento di Fisica, Università degli Studi di Bologna, V. Irnerio 46, Bologna, Italy \\ (Received 20 July 2008; published 26 February 2009)
}

\begin{abstract}
A few years ago, a novel multiturn extraction scheme was proposed, based on particle trapping inside stable resonances. Numerical simulations and experimental tests have confirmed the feasibility of such a scheme for low order resonances. While the third-order resonance is generically unstable and those higher than fourth order are generically stable, the fourth-order resonance can be either stable or unstable depending on the specifics of the system under consideration. By means of the normal form, a general approach to control the stability of the fourth-order resonance has been derived. This approach is based on the control of the amplitude detuning and the general form for a lattice with an arbitrary number of sextupole and octupole families is derived in this paper. Numerical simulations have confirmed the analytical results and have shown that, when crossing the unstable fourth-order resonance, the region around the center of the phase space is depleted and particles are trapped in only the four stable islands. A four-turn extraction could be designed using this technique.
\end{abstract}

DOI: 10.1103/PhysRevSTAB.12.024003

PACS numbers: 05.45.-a, 29.27.Bd

\section{INTRODUCTION}

A novel type of extraction based on particle trapping inside stable islands of the horizontal phase space was proposed [1-4] in 2003. In this scheme the beam manipulation is intrinsically linked with nonlinear beam dynamics. The beam is swept through a nonlinear resonance and, whenever the crossing is adiabatic, some particles can be trapped inside the stable islands and then transported towards higher amplitudes. At this stage, the beam extraction proper can be performed.

Such an extraction mode is primarily aimed at replacing the current continuous transfer (CT) extraction [5] from the CERN Proton Synchrotron (PS) to the Super Proton Synchrotron (SPS). The losses in the PS are highly reduced, if not completely suppressed, and injection matching in the SPS is improved. A detailed account of the expected optical mismatch at SPS injection for the CT extraction mode was presented in Ref. [6], based on the computation of equivalent optical parameters using the second-order moments of the final beam distribution after beam shaving (see, e.g., Ref. [7]).

A long measurement campaign was performed [8-12] to assess the performance of the newly proposed method, and a project was set up to implement it in the PS machine [13]. The new extraction method was commissioned in Summer 2008.

*diego.quatraro@cern.ch
Further studies, however, have shown that the method has a much broader scope, being applicable to resonances other than the $1 / 4$, which was the one selected for the CERN-specific application [4]. In addition, since the scheme can be time reversed, corresponding to a reversal of the direction of the resonance crossing, yet another new type of injection can be envisaged [14].

In the case of a stable resonance of order $n, n+1$ beamlets are generated, $n$ corresponding to the beam trapped into the stable islands and 1 to the beam core remaining after the trapping process. The $n+1$ beamlets at the end of the trapping process form two disconnected structures in phase space. The first is a ribbon closing up after $n$ turns around the machine and the second, at the center of phase space, closing up after one turn.

It is worth stressing that the longitudinal beam structure has no impact on the transverse splitting. In this respect, both bunched and continuous beam structures can be envisaged. Nonetheless, the longitudinal beam structure does have an impact on the extraction losses due to the finite rise time of the extraction kickers.

At the level of the beam extraction system proper a set of kickers generates a fast bump, constant over the $n+1$ extraction turns, noting that the kickers' strength must be increased for the last turn. This necessary increase might be a limiting factor of the scheme since it imposes higher demands on the strength requirements. An unstable resonance, for which there is basically no beam left at the center of phase space, could therefore be advantageous. 
The prototype for such an unstable behavior is the thirdorder resonance. Under a change of the betatronic tune approaching the resonant value, the unstable fixed points and the related separatrix shrink to zero. This implies that whenever the exact resonant value of the tune is achieved the closed orbit becomes unstable and particles in the neighborhood of the origin will be repelled and pushed towards higher amplitudes.

The case of the third-order resonance has been studied in [4]. Since the third-order resonance is generically unstable, there is no need to determine special conditions for the strength of nonlinear magnetic elements to empty the region around the origin of phase space during resonance crossing. In applications to real machines-such as the PS as SPS injector-the use of the third-order resonance would produce only a three-turn extraction, which would neither ensure a simultaneous optimization of the filling time of the SPS nor a reduction of the transient effects, such as beam loading in the SPS rf cavities.

Given these constraints, the fourth-order resonance should be considered as it could be made unstable so as to generate a four-turn extraction scheme, which would certainly be more effective. This, however, implies determining a strategy to change the stability type of the resonance, so that the separatrix related to the unstable fixed points collapses to the origin when the transverse tune is varied. The method proposed in this paper consists of applying perturbation theory to the model representing only the horizontal beam dynamics. This is justified as the proposed extraction requires manipulating the transverse beam distribution in only the horizontal plane. In fact, provided the nonlinear magnetic elements generating the stable islands are located in sections where the vertical beta functions [15] are small compared to the horizontal ones, the nonlinear coupling between the two transverse planes can be safely neglected. The correctness of such an approximation was based on the theoretical predictions described in Refs. [1,4] and tested repeatedly during experimental campaigns [8-12]. The Hamiltonian description of the nonlinear motion of a particle beam in a circular accelerator and its confirmation by experiment was described in a number of key papers reporting on studies performed at the Tevatron [16], and at the Indiana University Cyclotron Facility (IUCF) cooler ring [17]. The approach described in this paper is therefore based on solid theoretical and experimental foundations.

The properties of the dynamical system are best studied using the Hamiltonian function, as its structure permits the determination of the coefficients which rule the stability of the resonance as well as the free parameters, e.g., strength of the nonlinear magnetic elements, that can be best used to control the relevant terms in the Hamiltonian.

The best way to perform such an analysis is probably the normal form as it enables the generation of the so-called interpolating Hamiltonian starting from the polynomial one-turn map, which, for a circular accelerator, can be easily constructed for either the nonresonant or resonant or quasiresonant case [18] retaining the essential features of the original system. The literature on the normal form is wide, ranging from the mathematical papers on the foundations (see, e.g., Refs. [19-21]) to those dealing with applications to particle accelerators (see, e.g., Refs. [22$24]$ ), including the reviews $[18,25]$.

The detailed analysis of the problem and of the proposed solution is presented in Ref. [26]. In this paper, a brief summary of the approach will be presented in Sec. II together with a discussion on how to make a resonance unstable. The model used in the numerical simulations is described in Sec. III, while the results are discussed in Sec. IV. A by-product of this study is a potential solution to the issue of equally sharing the intensity between the five beamlets in the case of the stable fourth-order resonance; this aspect is also discussed at the end of Sec. IV. Finally, some conclusions are drawn in Sec. V. Some key results of the normal form theory are reported and discussed in Appendix A together with the proof of the main analytical results used in this study.

For the sake of completeness, it is worth pointing out that, while the use of nonlinearities to act on the transverse parameters of particle beams is rather new, manipulations in the longitudinal plane have been described in the literature during the 1990s (see, e.g., Ref. [27] where the longitudinal dynamic is used to induce amplitude growth in the transverse plane for crystal channeling).

\section{STABILITY CHANGE OF RESONANCES}

In this section, the proposed method of controlling the stability type of the transverse resonance is presented. As already mentioned in the Introduction, such an approach is based on normal form theory, whose features relevant to this study are reported in Appendix A. The starting point is a generic $2 \mathrm{D}$ symplectic polynomial map,

$$
\left(\begin{array}{c}
x \\
p_{x}
\end{array}\right)_{n+1}=R(\omega)\left(\begin{array}{c}
x \\
p_{x}+g_{N}(x)
\end{array}\right)_{n},
$$

where $\left(x, p_{x}\right)$ are the Courant-Snyder canonical coordinates [15], $R(\omega)$ is a rotation matrix, $\omega=2 \pi \nu$ is the linear frequency, and $g_{N}(x)$ is a polynomial function of order $N$ in $x$.

The idea behind the normal form is to construct a change of variables, defined by a polynomial function, in such a way that in the new coordinates the symmetries of the original system are immediately visible in the transformed map $U$ (the so-called normal form). The properties of the map $U$ are linked to those of the frequency $\omega$, and, in particular, whether it is rational or irrational. As the map $U$ is computed via a perturbative scheme, only a truncated approximation to the true function is available. However, the dynamics induced by the normal form cannot be studied using a truncation, as in general it is not symplec- 
tic, but it is possible to construct a time-independent Hamiltonian whose flow interpolates the phase space trajectories of $U$ at integer times. Such a Hamiltonian, called the interpolating Hamiltonian, is a constant of motion and the curves of constant energy interpolate the orbits of the normal form. The general form of this function is presented in Appendix A, while the details of the derivation can be found in Ref. [18]. For the purposes of this paper, the frequency will be assumed to be next to a rational value in order to simulate the resonance crossing under consideration. This situation corresponds to the so-called quasiresonant case, where $\omega=2 \pi(p / q+\eta)$, with $p, q \in \mathbb{Z}$, and $\eta \in \mathbb{R}$. Assuming $q=4$ (the actual value of $p$ is not relevant for this form of the Hamiltonian), then the dynamic is governed by

$H(\vartheta, \rho)=\eta \rho+\Omega_{2} \rho^{2}+\mathcal{A} \rho^{2} \cos 4 \vartheta+\frac{\Omega_{4}}{3} \rho^{3}+\mathcal{O}\left(\rho^{3}\right)$,

where action-angle variables $(\vartheta, \rho)$ have been introduced (see Appendix A for some comments on the properties of $\rho$ ).

The essence of the dynamics of the original system can be extracted from such a Hamiltonian. In particular, the geometrical characteristics of the orbits, such as fixed points and their stability, separatrices, and nonlinear tune can be investigated. The terms independent on the angle are responsible for the change of frequency in phase space (the so-called detuning with amplitude). The terms dependent on the angles introduce a distortion of the phase space which gives rise to fixed points, both stable and unstable. The latter are linked by separatrices, which can reach the origin of phase space for $\eta=0$, thus changing the stability type and making it unstable. Therefore, particles are repelled towards higher amplitudes, where the stable islands can eventually trap them when the resonance is crossed.

By inspecting Eq. (2), the strategy to obtain a change of the stability type of the origin requires setting the coefficient $\Omega_{2}$ to zero. By doing so, the lowest-order term in $\rho$ is the resonant one when $\eta \rightarrow 0$. In Appendices B, C, and D the details of the computations of the nonresonant term $\Omega_{2}$ are reported taking into account not only its dependence on the strength of the nonlinear elements, but also the phase advance between them. This information is used to control $\Omega_{2}$ during the resonance crossing process.

It is worthwhile pointing out that the proposed approach to changing the stability type of the fourth-order resonance can be generalized to higher-order resonances as well. In fact, from the general form of the interpolating Hamiltonian (reported in Appendix A) in Eq. (A21) it is possible to show that whenever all nonresonant terms up to order $q / 2$ in $\rho$ are set to zero, then the order $q$ resonance becomes unstable, as all the $\rho$-dependent terms are zero and the first nonzero term for $\eta=0$ is the resonant one. Although possible on paper, such an approach would hardly be feasible in a real machine. Indeed, it can be shown (see Ref. [18]) that the terms $\Omega_{2 j}, j>1$ depend linearly on the strength of high-order magnets (decapoles or higher) and nonlinearly on the strength of sextupoles and octupoles. The use of high-order nonlinear magnets has the advantage of reducing the conditions $\Omega_{2 j}=0,1 \leq$ $j \leq q / 2$ to a set of linear equations. On the other hand, the challenge is then shifted to the construction of such magnets. Alternatively, the use of families of sextupole and octupole magnets relies on finding a robust solution to the system of $q / 2$ highly nonlinear equations, which, while certainly possible on paper, seems rather unlikely for a real machine.

\section{MODEL}

The model chosen for studying the adiabatic capture process consists of the composition of two polynomial symplectic maps, namely,

$$
\begin{aligned}
& \left(\begin{array}{c}
x \\
p_{x}
\end{array}\right)_{n+1}=R\left(\psi_{2}\right)\left(\begin{array}{c}
x \\
p_{x}+k_{3,2} x^{3}
\end{array}\right) \circ \\
& R\left(\psi_{1}\right)\left(\begin{array}{c}
x \\
p_{x}+k_{2,1} x^{2}+k_{3,1} x^{3}
\end{array}\right)_{n},
\end{aligned}
$$

where $\left(x, p_{x}\right)$ are Courant-Snyder phase space coordinates, $R(\varphi)$ is a rotation matrix of an angle $\varphi, k_{2,1}, k_{3,1}$, and the operator $\circ$ represents the composition of functions. The strengths of one sextupolar and two octupolar elements are indicated with $k_{3,2}$, respectively. They are defined as

$$
k_{n}=\frac{L}{(B \rho)} \frac{d^{n} B_{y}}{d x^{n}},
$$

where $L$ is the length of the magnetic element, $(B \rho)$ is the magnetic rigidity, and $B_{y}$ is the vertical component of the magnetic field. Without any loss of generality the sextupolar strength can be set to one, as it is always possible to define rescaled coordinates such that the coefficient of the quadratic term in Eq. (3) equals one $[4,18]$ and the $k_{3, i}$ are also transformed into $\hat{k}_{3, i}$. It is worth emphasizing that the new rescaled coordinates are dimensionless and such a rescaled map is used for all numerical simulations presented in this paper.

The underlying physical model is a circular machine with a set of sextupoles and octupoles located at the same longitudinal position and a second octupole located at a different position. Two independent octupoles are needed to act on the coefficient $\Omega_{2}$ and, at the same time, to change the stable islands' parameters. This would also be the minimal requirement in view of an experimental test, e.g., at the CERN PS. During the iteration of the map the value of $\psi_{1}$ is kept constant, letting $\psi_{2}$ vary with $n$, so as to set $\omega=2 \pi \nu=\psi_{1}+\psi_{2}$ to the actual value of the linear frequency of the system. 
As already mentioned, the computation of the nonresonant term $\Omega_{2}$ required for the resonance stability control is shown in Appendices B, C, and D, indeed for a more general case than the map (3). In fact, the composition of $M$ polynomial maps of the form

$$
\left(\begin{array}{c}
x \\
p_{x}
\end{array}\right)_{n+1}=R\left(\psi_{j}\right)\left(\begin{array}{c}
x \\
p_{x}+k_{2, j} x^{2}+k_{3, j} x^{3}
\end{array}\right)_{n}
$$

is considered, and the model describes a machine where $M$ sextupoles and octupoles are installed reflecting the configuration of the CERN PS machine. Each main dipole features a set of additional coils, the so-called pole face windings and the figure-of-eight loop, that generate higherorder components to enable control of the machine working point (see Refs. [28,29] for more details and Fig. 1 for a sketch of the PS main magnet together with the configuration of the additional coils). It turns out that these components include sextupolar as well as octupolar magnetic multipoles [30]. This more realistic model is used here only to derive the general expressions for $\Omega_{2}$ as a function of the nonlinear parameters of the system, but not for numerical simulations. Clearly for $M$ maps, $\omega=$ $\sum_{i=0}^{M} \psi_{i}$ is the global tune.

For the rescaled version of the map (3) the condition $\Omega_{2}=0$ corresponds to

$$
3 \cot \left(\frac{\omega}{2}\right)+\cot \left(\frac{3 \omega}{2}\right)+6\left(\hat{k}_{3,1}+\hat{k}_{3,2}\right)=0,
$$

as a consequence of Eq. (D4). Therefore, the strength of the nonlinear elements can be either a function of $\omega$, such that $\Omega_{2}$ is set to zero throughout the whole resonance crossing process, or a constant such that $\Omega_{2}=0$ is fulfilled only for the resonant value of the tune. For the model used in the numerical simulations the values $\psi_{1} / 2 \pi=0.62, \hat{k}_{3,1}=1$, and $\hat{k}_{3,2}=-4 / 3$ were used, retaining the option of constant nonlinear strengths.

A typical sequence of phase space topology obtained during the resonance crossing process is shown in Fig. 2. By changing the tune, the separatrices related to the hyperbolic fixed points shrink towards the origin, making the resonance unstable.

\section{NUMERICAL SIMULATIONS}

The numerical simulations were performed using the model of Eq. (3) using a Gaussian distribution of particles

$$
\rho\left(x, p_{x}\right)=\frac{1}{2 \pi \sigma^{2}} e^{-\left(x^{2}+p_{x}^{2}\right) /\left(2 \sigma^{2}\right)}, \quad \sigma^{2}=\epsilon_{x, 0}
$$

with $\epsilon_{x, 0}$ the emittance of the initial Gaussian distribution in the normalized coordinate system. The tune $\nu(n)$ is changed with time as a polynomial function $f_{k}$ of order $k$, where the key parameters are $\nu_{s}, \nu_{r}$, and $\nu_{f}$, the starting, resonant, and final tune values, respectively. The turn at which the resonance is crossed and the total number of turns involved in the capture process are indicated by $n_{1}$, and $N$. The order of the polynomial might be changed

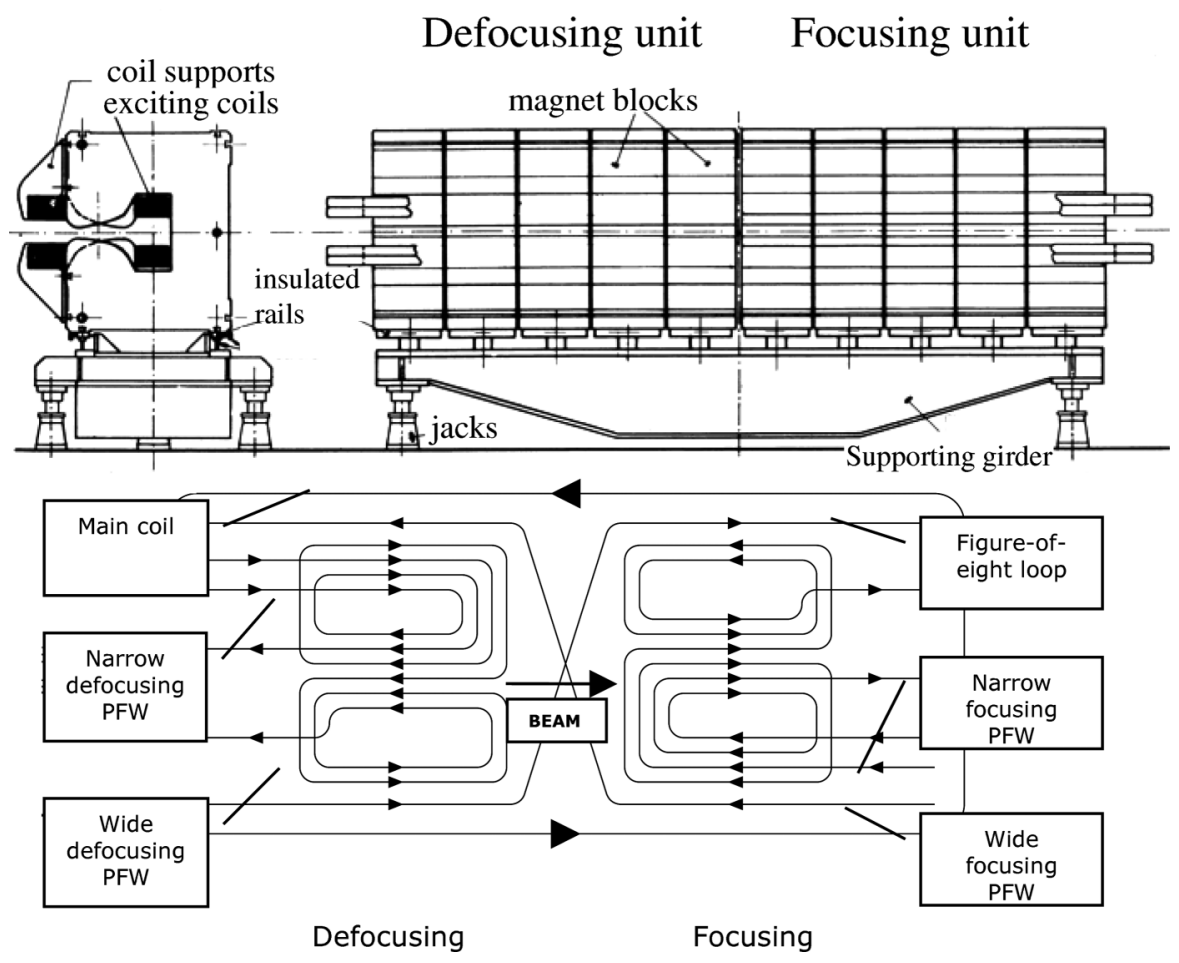

FIG. 1. Sketch of PS main magnet (upper) and of the five circuits used to control the tunes and the chromaticities (lower). 

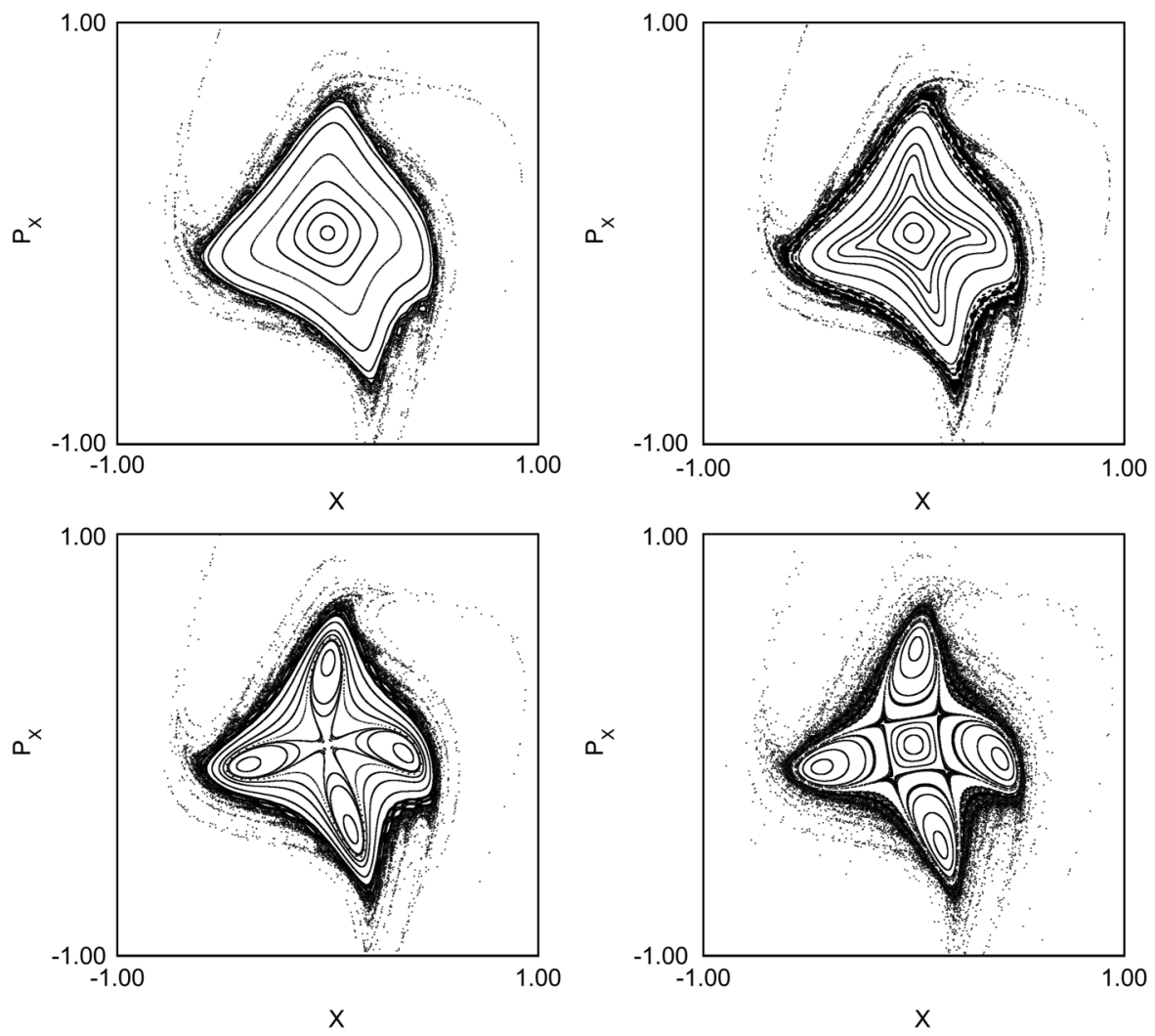

FIG. 2. Phase portrait of the dynamical system described by Eq. (3) as the linear tune $\nu$ is changed and approaching the resonant value $\nu=1 / 4$ from above.
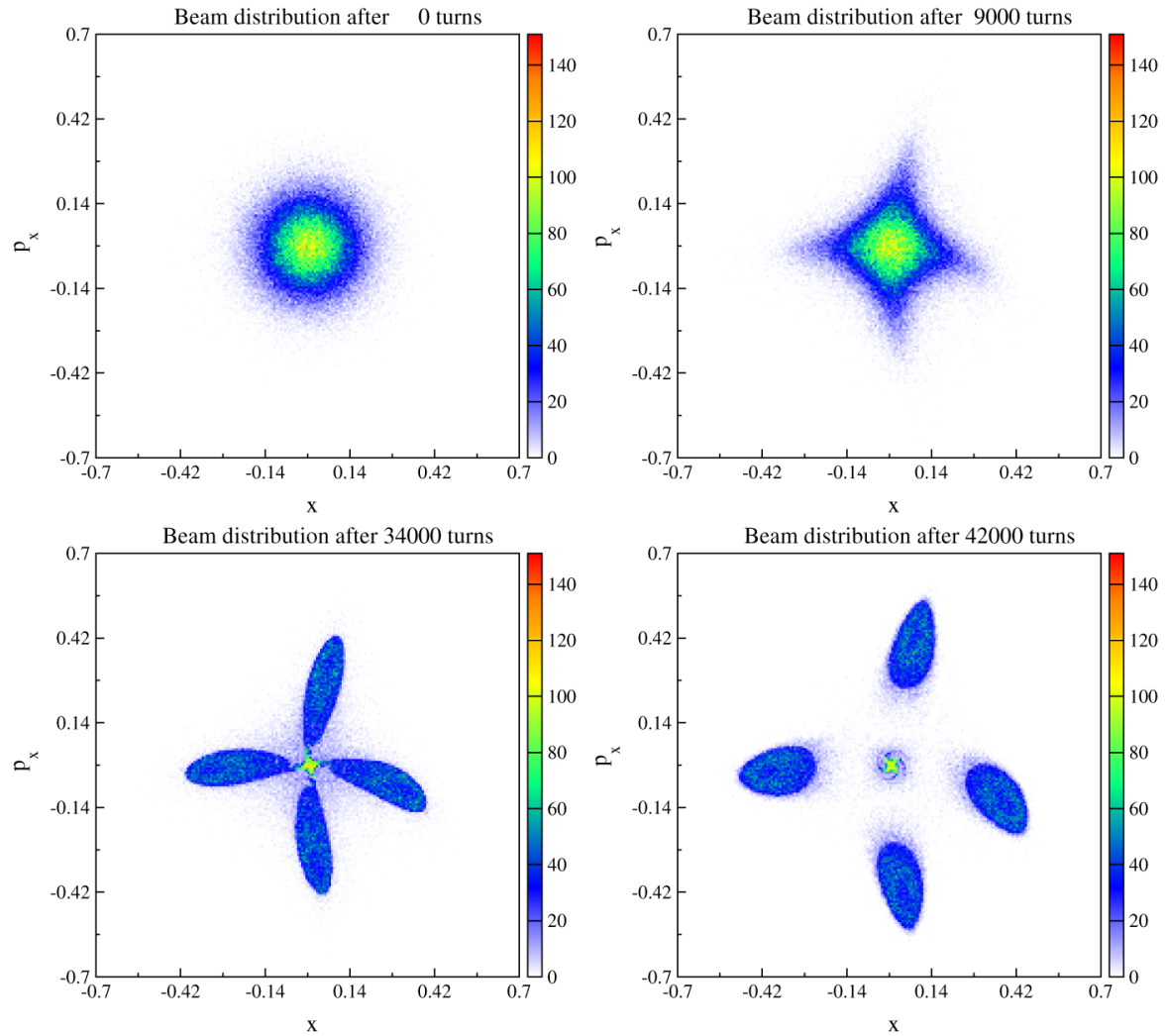

FIG. 3. (Color) Evolution of the initial distribution during the resonance crossing process. 


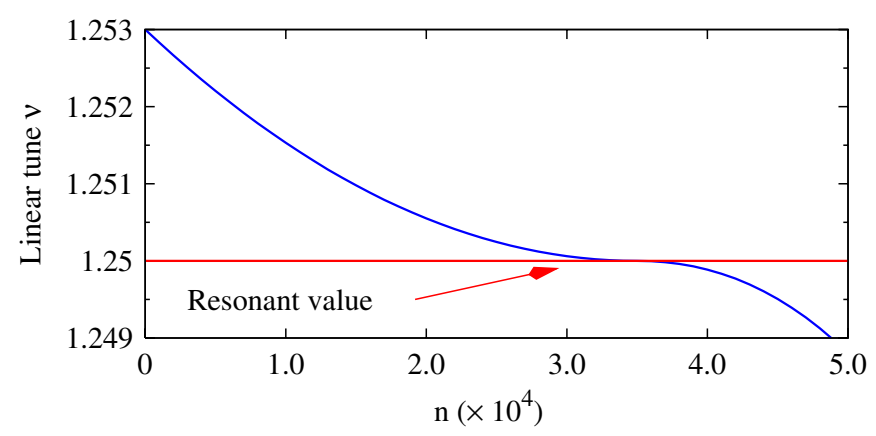

FIG. 4. (Color) Tune variation used for the splitting presented in Fig. 3. The tune curve is of type $2-3$.

before and after $n_{1}$ with the constraint, however, that $\nu^{\prime}\left(n_{1}\right)=0$ in order to achieve a smooth transition at the exact resonance crossing. Henceforth, we refer to an $i-j$ curve if the polynomial curve is of the $i$ th order before the resonance crossing and of the $j$ th order afterwards.
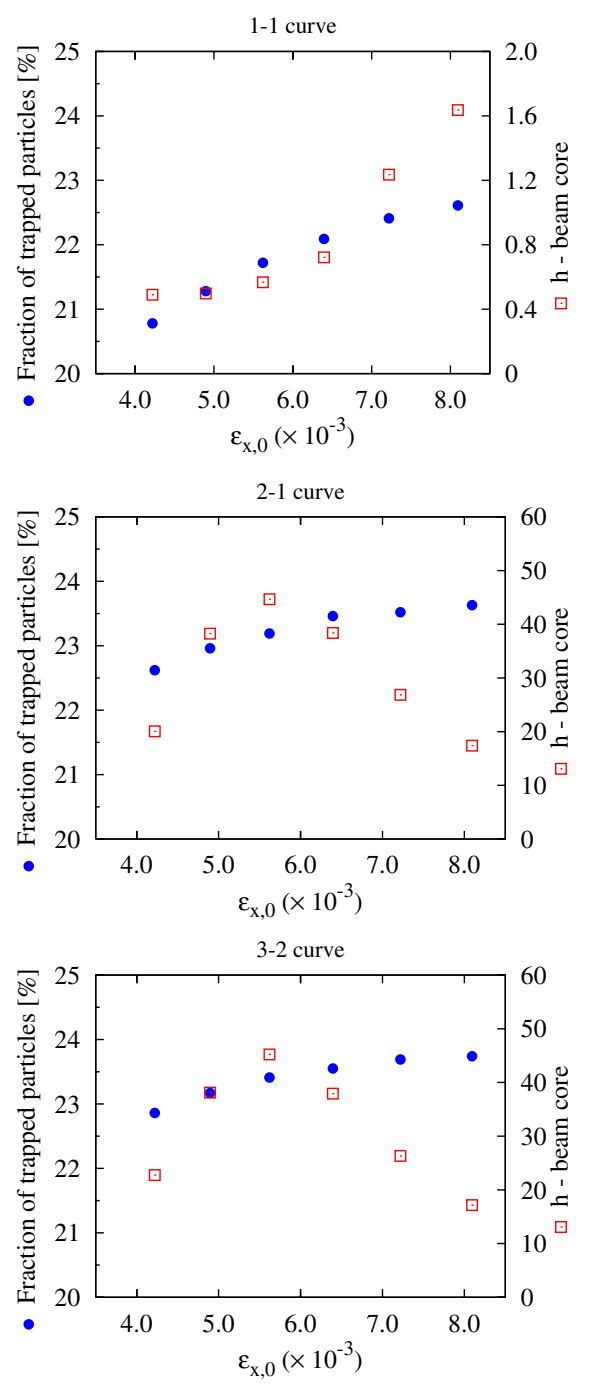

Typically, $2 \times 10^{6}$ particles are tracked for about $5 \times$ $10^{4}$ iterations of the map (turns), with $\nu_{s}=1.253, \nu_{r}=$ 1.25 , and $\nu_{e}=1.248$, where the resonance crossing occurs for $n_{1}=3.5 \times 10^{4}$. An example of the splitting process is shown in Fig. 3 and the corresponding tune variation is reported in Fig. 4. While the separatrices collapse towards the origin, when the tune is varied towards the resonant value, the particles are pushed towards the stable islands and trapped inside. At the very end of the process (lower left plot), the islands are also moved towards higher amplitudes. During the whole process no particle loss is observed. While no detailed aperture model is used in the numerical simulations every particle's coordinate is checked at each turn and, whenever it is outside the square $[-1,1] \times[-1,1]$ in the phase space, the particle is considered lost.

The particles left near the origin of phase space are perturbed by the separatrix crossing and the final distribution is far from the initial Gaussian. Furthermore, around
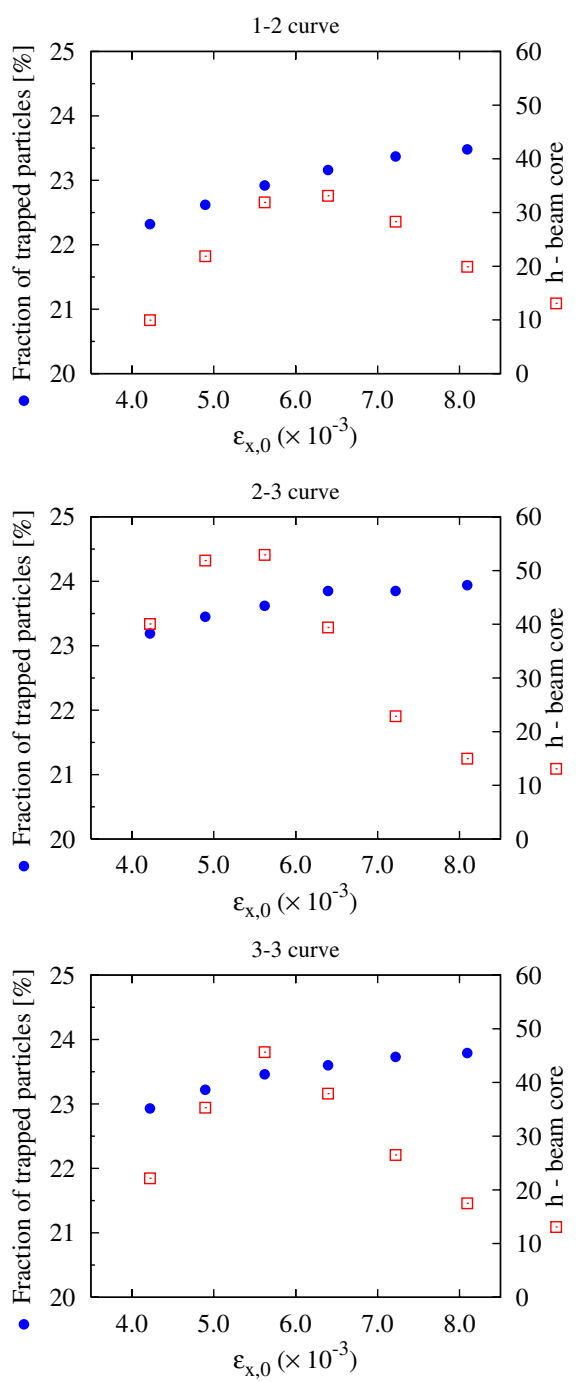

FIG. 5. (Color) Fraction of trapped particles as a function of the initial emittance. Six types of tune curves are used (marked on top of each plot). The value of the factor $h$ for the beam core after splitting is also given. 
the origin the adiabatic condition tends to be violated as the nonlinear frequency of motion tends to zero. Therefore, the fraction of particles left there is very sensitive to the time dependence of the tune.

In Figs. 5 and 6 the fraction of the trapped particles in the resonance islands and their relative emittances $\epsilon_{x} / \epsilon_{x, 0}$ are, respectively, shown as functions of the emittance $\epsilon_{x, 0}$ of the initial Gaussian distribution equation (7). As the curves are the same for the four islands, only one is shown in the plot. For the particles remaining close to the origin of the phase space, the so-called halo parameter $h$ [31] defined as

$$
h=\frac{\left\langle x^{4}\right\rangle}{\left\langle x^{2}\right\rangle^{2}}-2
$$

is shown in Fig. 5. The bigger the $\sigma$ the greater is the fraction of beam trapped in the resonance islands. This is true for every type of tune curve, even though the overall variation of the fraction of trapped particles versus the emittance of the initial distribution is not large. The figure also shows clearly that the higher the order of the polynomial the smaller is the fraction of beam left in the center. For the curve $1-1, \approx 10 \%$ of the beam is left in the core distribution for the largest emittance. The best result is obtained for the curve $2-3$ with a fraction of $\approx 4 \%$ remaining. The huge value of the halo parameter indicates that what is left at the origin is strongly perturbed and the distribution features very heavy tails.

The final beamlets emittance decreases steadily with increasing initial emittance, and also with the adiabaticity of the tune curve. The smoother the resonance crossing, the smaller is the relative beamlet emittance. In all cases, however, the final beamlet emittance is never smaller than one-half of the initial value. This indicates that the interaction between the beam distribution and the separatrix is inducing some emittance blowup. Therefore, the
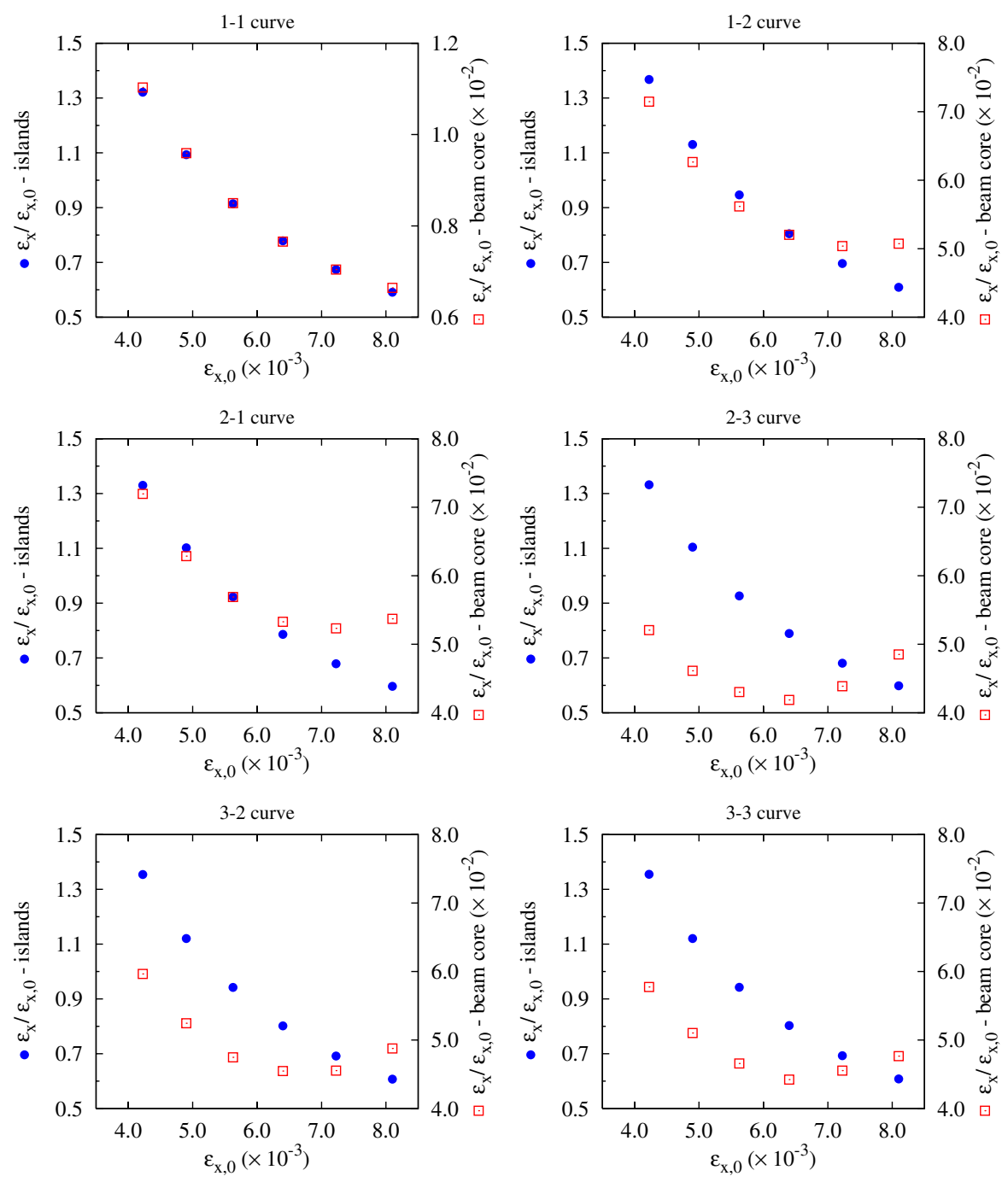

FIG. 6. (Color) Relative islands emittance as a function of the initial emittance. Six types of tune curves are used (marked on top of each plot). 

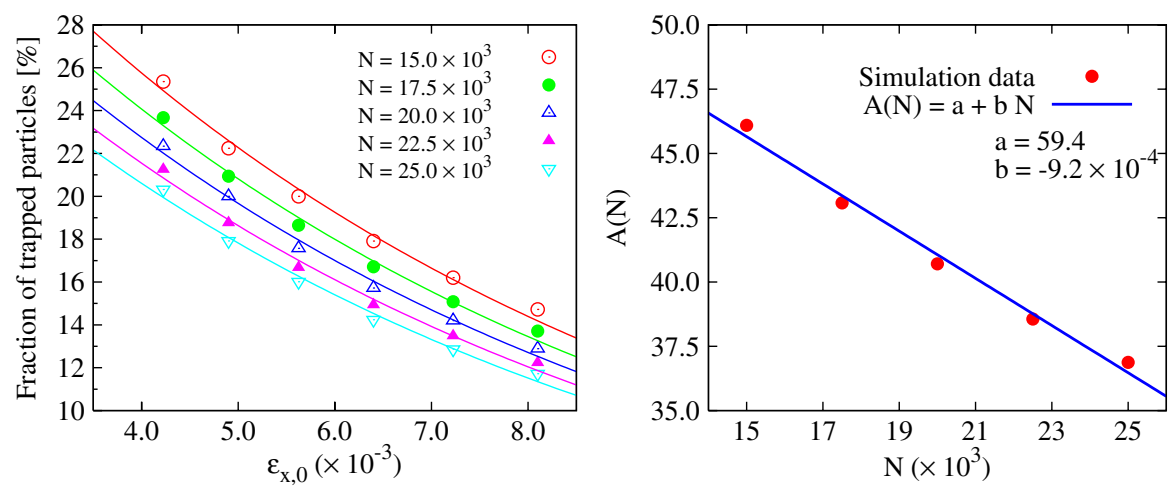

FIG. 7. (Color) Fit results of the fraction of trapped particles as a function of the total number of turns $N$ with an exponential function (left). The amplitude of such an exponential is fitted as a linear function of $N$ (right).

expected reduction of the initial emittance by about a factor of 4 (due to the beam sharing in four islands) does not seem to be possible.

A set of numerical simulations was performed to assess the dependence of the fraction of trapped particles on the total turn number $N$ over which the tune variation $\Delta \nu=$ $\nu_{f}-\nu_{i}$ is performed and the $\sigma$ of the initial distribution. A tune curve of type $1-1$ was used for this study. The dependence on $\sigma$ is perfectly fitted by an exponential function, while that on $N$ is a linear function and the combined result gives $N_{\text {core }} / N_{\text {part }}=A(N) e^{-c \sigma}$ with $A(N)=a+b N$, where $N_{\text {part }}$ stands for the total number of particles. The numerical results and the fitted curves are shown in Fig. 7. The excellent agreement between the data and the fit function is clearly visible. From the numerical simulations it is also clear that the parameter $c$ does not depend on $N$. Nevertheless it is still possible that the nonlinear parameters of the system, determining the islands' size and position, have an impact on the parameter $c$. The fit values are listed in Table I.

In the numerical simulations presented so far, the condition $\Omega_{2}=0$ was imposed only for the resonant value of the tune. Comparisons with simulations performed with $\Omega_{2}=0$ throughout the whole process showed a further decrease of the fraction of particles left in the center to $\approx 0.5 \%$. This shows that the intensity sharing is affected by the phase space topology mainly during the stage of resonance crossing, the rest of the tune variation serving only to displace the beamlets to higher amplitudes.

An additional test was performed to study the impact of setting $\Omega_{2}=\Delta$ with $\Delta$ a small positive quantity. The condition $\Omega_{2}=0$ is required for the separatrices to pass

TABLE I. Fit parameters for the scaling laws determined with the numerical simulations.

\begin{tabular}{lccc}
\hline \hline Relation & $a$ & $b$ & $c$ \\
\hline$N_{\text {core }} / N_{\text {part }}=(a+b N) e^{-c \sigma}$ & 131.16 & $-1.8 \times 10^{-3}$ & 22.26 \\
$N_{\text {core }}=a+b \Delta+c \Delta^{2}$ & 4.78 & -9.68 & 253.12 \\
\hline \hline
\end{tabular}

through the origin of phase space, thus making the resonance unstable. The whole process of beam splitting, however, relies not only on the topology of the phase space, but also on the way the time dependence is generated. The key issue is the adiabaticity of the process. It is clear that such a property is lost in a small neighborhood of the origin, as the particle's frequency tends to zero so that the tune variation can never be slow with respect to the transverse dynamics. This explains why some particles are always left almost unaffected by the resonance crossing. Therefore, it seems reasonable that a small deviation to the condition $\Omega_{2}=0$ should have a negligible impact on the final beam parameters. Furthermore, this numerical test could give indications of the accuracy required for controlling $\Omega_{2}$ in a experimental test. The figure of merit of this test is the fraction of particles left in the core, which is shown in Fig. 8. A parabolic behavior is clearly visible and it can be fitted very accurately. The minimum for $N_{\text {core }} / N_{\text {part }}$ is achieved for slightly positive values of $\Delta$. Again the fit values are listed in Table I.

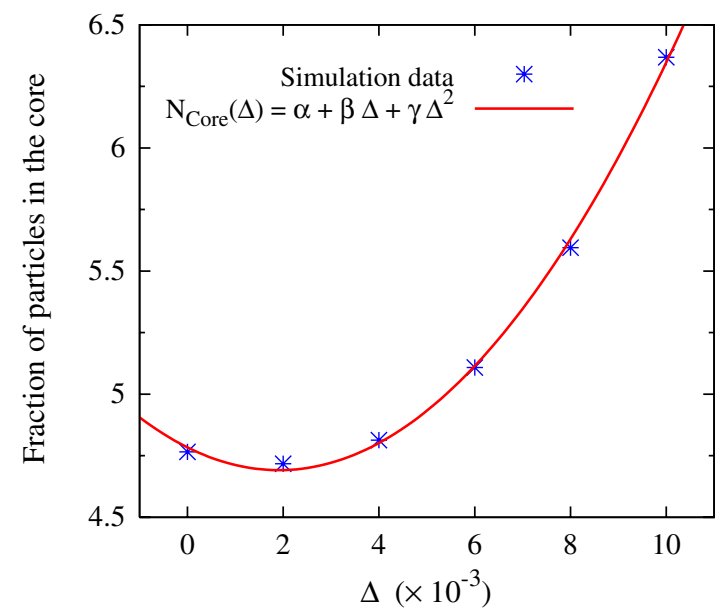

FIG. 8. (Color) Fraction of particles remaining in the beam core as a function of $\Delta$. The fitted parabola is also shown. The numerical simulations are performed with $\sigma=0.08$ for the initial distribution. 

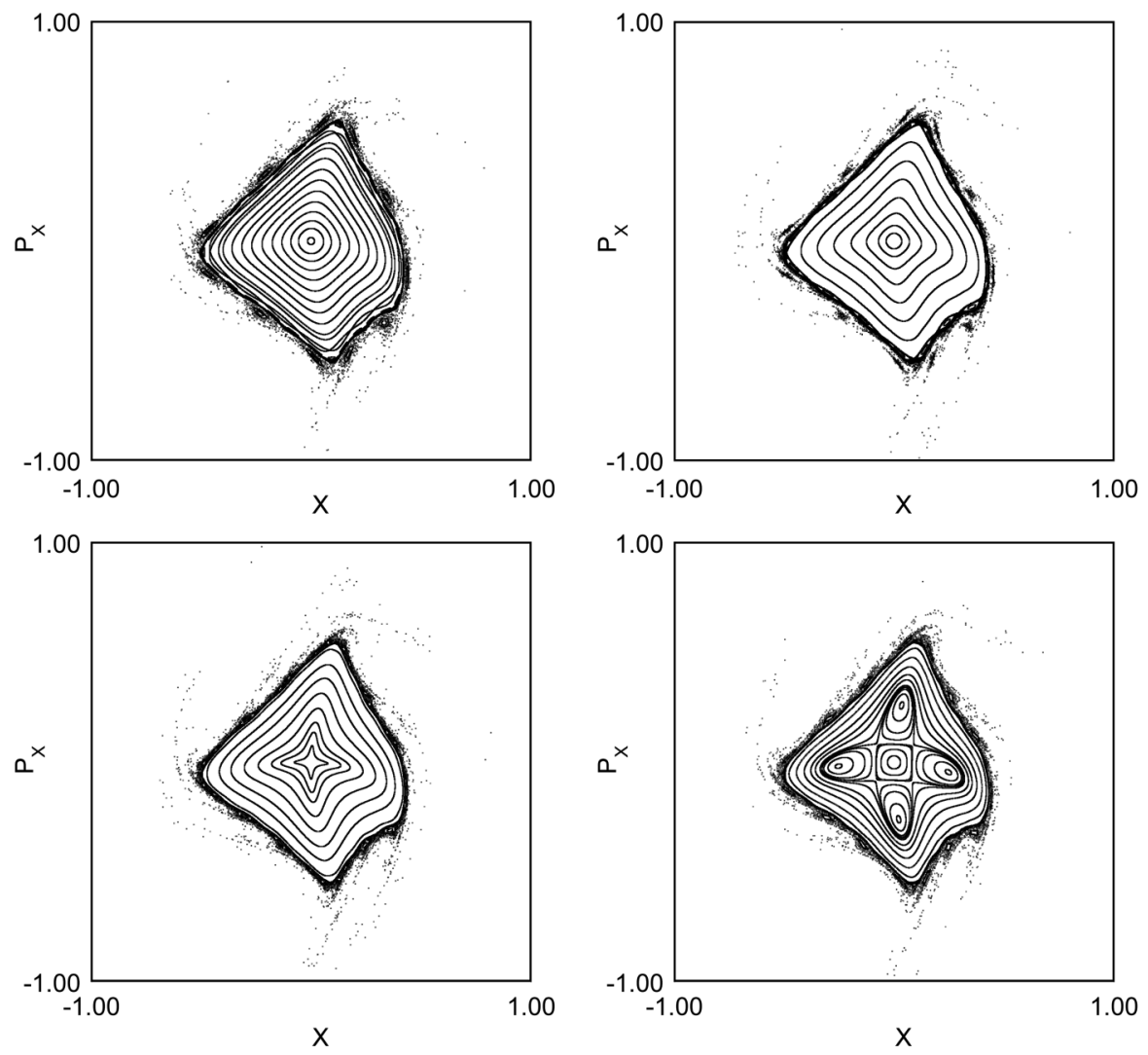

FIG. 9. Phase portrait of the dynamical system [Eq. (3)], corresponding to $\Delta=0.265$, as the linear tune $\nu$ is changed and approaching the resonant value $\nu=1 / 4$ from above.

Interestingly enough, one could use this scaling law to find the value of $\Omega_{2}$ for which the intensity is equally shared between the four beamlets and the center core. As already mentioned this is a crucial point for a five-turn extraction. From the fit results the $20 \%$ sharing is obtained for $\Delta \approx 0.265$. Numerical simulations confirmed this finding showing that for such a value of $\Delta$ and for an initial $\sigma=0.08$ the actual sharing is $20.3 \%$ in the islands and $19.7 \%$ in the core with a tune curve of type $2-3$. The corresponding phase space portrait is shown in Fig. 9. This result confirms that the analytical approach can shed some light on the detail of the beam splitting and that it can be extremely useful for the overall optimization of the process. In particular, it will be used for the definition of the optimal parameters values in the case of a five-turn extraction with the aim of achieving a perfect sharing of the intensity among the various beamlets.

\section{CONCLUSIONS}

In this paper the use of the normal form to study the change of the stability type of a nonlinear resonance was presented. A general framework was considered so that the proposed approach could be applied not only to a simplified model representing the 2D nonlinear betatronic motion, but also to a more realistic one with a set of $M$ sextupoles and octupoles. The more realistic model was defined taking into account the characteristics of the CERN PS machine.

Numerical simulations have shown that the fourth-order resonance can indeed be turned into an unstable resonance. This allows the center of phase space to be emptied while slowly varying the tune through the resonant value. The feasibility of a four-turn extraction design based on beam trapping in stable islands is therefore confirmed.

The dependence of the final beam parameters of the four generated beamlets on the type of resonance crossing was studied in detail. Different types of tune curves were tested and a clear dependence of the fraction of particles left in the center of the phase space on the exponent type was observed. The dependence on the sigma of the initial beam distribution was also studied. Furthermore, for a linear sweep through the resonance, the functional dependence of the final beam distribution on the crossing speed was derived.

Finally, the generalization of the condition used to make the fourth-order resonance unstable led to the interesting result that the control of the intensity sharing between the outer beamlets and the central core can be achieved by an appropriately chosen positive value of $\Omega_{2}$. Hence, during the resonance crossing, the sextupoles and octupoles should be controlled so as to provide the correct value of 
$\Omega_{2}$. This is of paramount importance for improving the performance of the proposed five-turn extraction being commissioned at the CERN PS.

\section{ACKNOWLEDGMENTS}

We would like to express our gratitude to A. Franchi and S. Gilardoni for many interesting and stimulating discussions on this topic. Furthermore, we would like to warmly thank E. McIntosh for proofreading the paper.

\section{APPENDIX A: NORMAL FORM THEORY}

In this Appendix, a brief review of the main concepts of the normal form theory is presented, limiting the description to the 2D case. The starting point is a generic symplectic polynomial map in the form of Eq. (1) using real Courant-Snyder canonical coordinates. It is customary to transform it to complex coordinates $\left(z, z^{*}\right)$ via the matrix $\mathbb{T}$

$$
\left(\begin{array}{c}
z \\
z^{*}
\end{array}\right)=\mathbb{T}\left(\begin{array}{c}
x \\
p_{x}
\end{array}\right)=\left(\begin{array}{cc}
1 & -i \\
1 & i
\end{array}\right)\left(\begin{array}{c}
x \\
p_{x}
\end{array}\right) .
$$

Then, the map reads

$$
z^{\prime}=F\left(z, z^{*}\right)=e^{i \omega} z+\sum_{n=2}^{N} \sum_{j=0}^{n} f_{j, n-j} z^{j} z^{* n-j},
$$

where

$$
f_{j, n-j}=-i e^{i \omega} \frac{n !}{2^{j} j !(n-j) !} g_{N, j}
$$

and $g(x)=\sum_{j=2}^{N} g_{N, j} x^{j}$ is the polynomial function representing the nonlinear component of the map (1), with $g_{N, 2}=1$.

The polynomial function defining the change of variables is such that in the new coordinates the symmetries of the original system are apparent in the transformed map $U$ (the so-called normal form). Formally, this is obtained by constraining the following diagram to commute

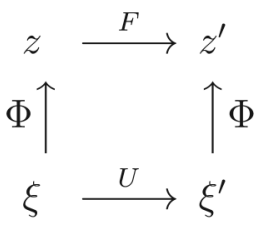

which corresponds to solving the functional equation,

$$
F \circ \Phi\left(\xi, \xi^{*}\right)=\Phi \circ U\left(\xi, \xi^{*}\right),
$$

where the operator $\circ$ represents the composition of functions, e.g.,

$$
F \circ \Phi\left(\xi, \xi^{*}\right)=F\left[\Phi\left(\xi, \xi^{*}\right), \Phi^{*}\left(\xi, \xi^{*}\right)\right] .
$$

The two unknown polynomial functions $\Phi$ and $U$ are expressed in terms of their coefficients as

$$
\begin{gathered}
z=\Phi\left(\xi, \xi^{*}\right)=\xi+\sum_{n \geq 2} \sum_{j=0}^{n} \phi_{j, n-j} \xi^{j} \xi^{* n-j}, \\
\xi^{\prime}=U\left(\xi, \xi^{*}\right)=e^{i \omega} \xi+\sum_{n \geq 2} \sum_{j=0}^{n} u_{j, n-j} \xi^{j} \xi^{* n-j},
\end{gathered}
$$

where $\Phi$ has been chosen tangent to the identity transformation. By solving Eq. (A5) order by order, the recurrence relations for $\phi_{j, n-j}, u_{j, n-j}$ are found, provided some additional constraints on the functional form of $U$ are imposed.

It is well known that the topology of the orbits of the system (1) depends on the properties of $\omega$. Two cases are possible.

(i) nonresonant if $\omega / 2 \pi$ is irrational.-Then the orbit is dense on a 1D torus (diffeomorphic to a circle) and $e^{i \omega}$ generates the symmetry group $\mathbb{U}(1)$ of continuous rotations.

(ii) resonant if $\omega / 2 \pi$ is a rational number $p / q$.-Then the orbit consists of a discrete set of $q$ points and the transformation $e^{i \omega}$ generates the discrete subgroup of $\mathbb{U}(1)$ of angles $2 \pi j / q$ with $j=0, \ldots, q-1$.

A map $U$ is in normal form if it is invariant with respect to a group, usually generated by its linear part. This condition has to be satisfied by each monomial $\xi^{n} \xi^{* m}$ of $U$. More explicitly, for a monomial of order $n$ such a condition reads

$$
e^{i(\omega+2 \pi k)} \xi^{j} \xi^{* n-j}=e^{i \omega j} e^{-i \omega(n-j)} \xi^{j} \xi^{* n-j}
$$

with $k \in \mathbb{Z}$. Thus, the solution is given by

$$
\vec{\omega} \cdot \vec{k}=0 .
$$

Assuming $\quad \vec{\omega}=(\omega, 2 \pi)$ and $\vec{k}=(2 j-n-1,-k)$, Eq. (A9) can be satisfied in two ways.

(i) When $\omega$ is nonresonant, then

$$
\vec{\omega} \cdot \vec{k}=0 \Leftrightarrow \vec{k}=0 .
$$

At order $n$ the monomials of $U\left(\xi, \xi^{*}\right)$ are only those of type

$$
\xi^{j} \xi^{* n-j}
$$

and $U$ reads

$$
\xi^{\prime}=e^{i \omega} \xi+u_{2,1} \xi^{2} \xi^{*}+u_{3,2} \xi^{3} \xi^{* 2}+u_{4,3} \xi^{4} \xi^{* 3}+\mathcal{O}\left(\xi^{7}\right) .
$$

Therefore, it is possible to express the nonresonant dynamics in terms of an amplitude-dependent rotation. If the action-angle variables $(\vartheta, \rho)$ are introduced, where

$$
\xi=\sqrt{\rho} e^{i \vartheta} \rightarrow \rho=\xi \xi^{*},
$$

the normal form can be rewritten as

$$
\xi^{\prime}=U\left(\xi, \xi^{*}\right)=e^{i \Omega(\rho)} \xi,
$$

where $\Omega(\rho)$ represents the frequency as a function of the action variable and it can be expanded in a power series as 


$$
\Omega(\rho)=\omega+\sum_{i \geq 1} \Omega_{2 j} \rho^{j} .
$$

It is worth stressing that, in the original coordinates, $\rho$ reads

$$
\rho=x^{2}+p_{x}^{2}+\text { higher order terms }
$$

which is obtained by using the definition of $\rho$ and Eq. (A7). Hence, $\rho$ corresponds to the Courant-Snyder invariant at the lowest order.

By replacing the expansion (A15) in Eq. (A14), developing the exponential in powers of $\rho$, and comparing order by order the analogous result obtained by transforming in action-angle variables [Eq. (A12)], it is found that

$$
\Omega_{2}=-i e^{-i \omega} u_{2,1} .
$$

(ii) When $\omega=2 p / q$ is resonant, then

$$
\vec{\omega} \cdot \vec{k}=0 \Leftrightarrow \vec{k}=l(q,-p),
$$

where $l$ is an arbitrary integer. In this case the monomials in normal form depend on the resonance under consideration. For the fourth order, $q=4$ and $p=1$ and the condition to be fulfilled reads $(2 j-n-1,-k)=l(4,-1)$. For each order $n$ the monomials satisfying the previous condition can be found and, up to order three, $U$ reads

$$
\xi^{\prime}=e^{i \omega} \xi+u_{2,1} \xi^{2} \xi^{*}+u_{0,3} \xi^{* 3}+\mathcal{O}\left(\xi^{4}\right) .
$$

It is worth noting that the term $u_{2,1}$ is common between the nonresonant and resonant case, while only the term $u_{0,3}$ is characteristic of the resonant normal form.

The computation of the term $u_{2,1}$ is crucial to the method presented in this paper, as it is proportional to $\Omega_{2}$, which is used to vary the stability type of the resonance. Indeed, it can be computed by using the fundamental equation (A5), truncating it at order 3, and selecting all terms of the form $\xi^{2} \xi^{*}$. After some algebra, one finds

$$
u_{2,1}=f_{2,1}+2 f_{2,0} \phi_{1,1}+f_{1,1}\left(\phi_{1,1}^{*}+\phi_{2,0}\right)+2 f_{0,2} \phi_{0,2}^{*},
$$

which is the main result used in the following Appendices.

For the quasiresonant case the dynamics induced by $U\left(\xi, \xi^{*}\right)$ cannot be studied using a truncation as it is not symplectic. To overcome this, it is possible to construct a time-independent Hamiltonian flow that interpolates the phase space trajectories of $U$ at integer times. Such an interpolating Hamiltonian is a constant of motion and its level lines interpolate the orbits of $U\left(\xi, \xi^{*}\right)$. The details concerning the derivation of this function can be found in Ref. [18]. For the aim of the study presented in this paper it is enough to report the general form of the quasiresonant Hamiltonian, which when transformed to action-angle variables reads

$$
\begin{aligned}
H(\vartheta, \rho)= & \eta \rho+\sum_{n=1}^{[(q-1) / 2]} \frac{\Omega_{2 n}}{n+1} \rho^{n+1} \\
& -\frac{\eta e^{-i \omega}}{e^{-i \eta q}-1} u_{0, q-1} \rho^{q / 2} e^{i q \vartheta} \\
& -\frac{\eta e^{i \omega}}{e^{i \eta q}-1} u_{0, q-1}^{*} \rho^{q / 2} e^{-i q \vartheta},
\end{aligned}
$$

where $q$ is the order of the resonance and [.] stands for the integer part of a real number. It is worth noting that $H(\vartheta, \rho)$ is continuous in the limit $\eta \rightarrow 0$, and in this case the features of the resonant case are recovered.

For the fourth-order resonance, $q=4$ and the expression (A21) reduces to (2), with the coefficient $\mathcal{A}$ given by

$$
\mathcal{A}=-2 \eta \mathfrak{R}\left[\frac{e^{i \omega}}{e^{i 4 \eta}-1} u_{0,3}^{*}\right] .
$$

\section{APPENDIX B: ANALYTICAL COMPUTATION OF THE COMPOSITION OF A SET OF SEXTUPOLAR MAPS}

In this Appendix the analytical form for the composition of $M$ quadratic maps, representing a machine with linear elements and $M$ sextupoles, is derived. By using the singlekick approximation [18] and assuming the use of normalized Courant-Snyder coordinates [15], the 2D map from the $j$ th to the $(j+1)$ th sextupolar element reads

$$
\left(\begin{array}{c}
x^{\prime} \\
p_{x}^{\prime}
\end{array}\right)=R\left(\psi_{j+1}\right)\left(\begin{array}{c}
x \\
p_{x}+k_{2, j} x^{2}
\end{array}\right) .
$$

The 2D matrix $R\left(\psi_{j+1}\right)$ represents a rotation of an angle $\psi_{j+1}$, the relative phase advance between the two elements, and $k_{2, j}=K_{2, j} \beta_{x, j}^{3 / 2} / 2$ is the strength of the sextupole weighted by the value of the optical beta function at the location of the nonlinear element (see also Ref. [4]). The arrangement of the set of sextupoles around the machine circumference is shown in Fig. 10, where $k_{n, j}$ stands for the coefficient of the $j$ th nonlinear $2(n+1)$-polar element. The computation of the coefficient $\Omega_{2}$ requires truncating the perturbative series to the third order according to Eqs. (A12), (A17), and (A20).

Assuming that $\psi_{0}=0$ and with the simplified notation $k_{j}=k_{2, j}$ and $\Psi_{n}=\sum_{i=0}^{n} \psi_{i}$ it is possible to prove the following.

Theorem 1.-The composition of $M$ quadratic maps of type (B1) in complex coordinates $\left(z, z^{*}\right)$ is given, up to third order, by 


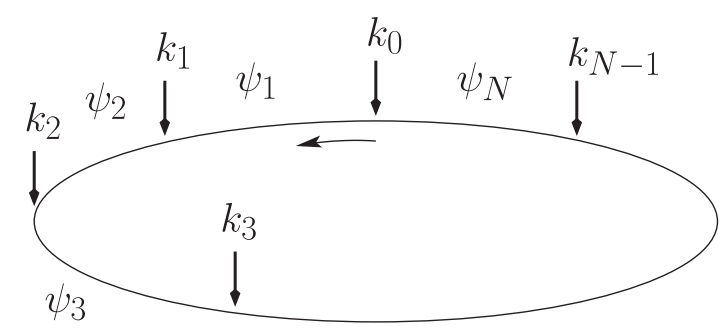

FIG. 10. Sketch of a machine circumference with the arrangement of several nonlinear lattice elements representing either sextupoles or octupoles.

$$
\begin{aligned}
z^{\prime}= & e^{i \omega}\left\{z-\frac{i}{4} \sum_{n=0}^{N-1} k_{n} \exp \left(-i \Psi_{n}\right)\right. \\
& \times\left[\exp \left(i \Psi_{n}\right) z+\exp \left(-i \Psi_{n}\right) z^{*}\right]^{2} \\
& -\frac{i}{4} \sum_{m=0}^{N-1} k_{m} \sum_{j=0}^{m} k_{j} \exp \left(-i \Psi_{m}\right) \sin \left(\Psi_{m}-\Psi_{j}\right) \\
& \cdot\left(\exp \left(i \Psi_{m}\right) z+\exp \left(-i \Psi_{m}\right) z^{*}\right) \\
& \left.\times\left[\exp \left(i \Psi_{j}\right) z+\exp \left(-i \Psi_{j}\right) z^{*}\right]^{2}\right\}
\end{aligned}
$$

Proof.-The statement is proved by induction. The proposition $P_{1}$ is trivially true. Then, assuming the propo- sition $P_{N}$ holds true, it will be shown that $P_{N+1}$ is true. This requires the computation of

$$
P_{N+1}=e^{i \psi_{N+1}}\left(z-\frac{i}{4} k_{N}\left(z+z^{*}\right)^{2}\right) \circ P_{N}
$$

which reads

$$
P_{N+1}=e^{i \psi_{N+1}} P_{N}-\frac{i}{4} k_{N} e^{i \psi_{N+1}}\left[\left(\left[P_{N}\right]_{\leq 2}+\left[P_{N}\right]_{\leq 2}^{*}\right)^{2}\right]_{\leq 3}
$$

where $[\cdot]_{j}$ represents the truncation of a polynomial at $j$ th order. It is seen that

$$
\begin{aligned}
{\left[P_{N}\right]_{\leq 2}=} & e^{i\left(\omega-\psi_{N+1}\right)}\left\{z-\frac{i}{4} \sum_{n=0}^{N-1} k_{n} \exp \left(-i \Psi_{n}\right)\right. \\
& \left.\times\left[\exp \left(i \Psi_{n}\right) z+\exp \left(-i \Psi_{n}\right) z^{*}\right]^{2}\right\} \\
{\left[P_{N}\right]_{\leq 2}^{*}=} & e^{-i\left(\omega-\psi_{N+1}\right)}\left\{z^{*}+\frac{i}{4} \sum_{n=0}^{N-1} k_{n} \exp \left(i \Psi_{n}\right)\right. \\
& \left.\times\left[\exp \left(i \Psi_{n}\right) z+\exp \left(-i \Psi_{n}\right) z^{*}\right]^{2}\right\}
\end{aligned}
$$

This implies also

$$
\begin{aligned}
{\left[P_{N}\right]_{\leq 2}+\left[P_{N}\right]_{\leq 2}^{*}=} & \left.\left(e^{i\left(\omega-\psi_{N+1}\right)} z+e^{-i\left(\omega-\psi_{N+1}\right)} z^{*}\right)-\frac{i}{4} e^{i\left(\omega-\psi_{N+1}\right.}\right) \sum_{n=0}^{N-1} k_{n} \exp \left(-i \Psi_{n}\right)\left[\exp \left(i \Psi_{n}\right) z+\exp \left(-i \Psi_{n}\right) z^{*}\right]^{2} \\
& +\frac{i}{4} e^{-i\left(\omega-\psi_{N+1}\right.} \sum_{n=0}^{N-1} k_{n} \exp \left(i \Psi_{n}\right)\left[\exp \left(i \Psi_{n}\right) z+\exp \left(-i \Psi_{n}\right) z^{*}\right]^{2} \\
= & \left(e^{i\left(\omega-\psi_{N+1}\right)} z+e^{-i\left(\omega-\psi_{N+1}\right)} z^{*}\right)+\frac{1}{2} \sum_{n=0}^{N-1} k_{n} \sin \left(\omega-\psi_{N+1}-\Psi_{n}\right)\left[\exp \left(i \Psi_{n}\right) z+\exp \left(-i \Psi_{n}\right) z^{*}\right]^{2}
\end{aligned}
$$

This result can be used to evaluate the truncation up to third order of the quadratic part of the maps, which is essential to the computation of the composition of the $N+1$ maps

$$
\begin{aligned}
{\left[\left(\left[P_{N}\right]_{\leq 2}+\left[P_{N}\right]_{\leq 2}^{*}\right)^{2}\right]_{\leq 3}=} & \left(e^{i\left(\omega-\psi_{N+1}\right)} z+e^{-i\left(\omega-\psi_{N+1}\right)} z^{*}\right)^{2}+\sum_{n=0}^{N-1} k_{n} \sin \left(\omega-\psi_{N+1}-\Psi_{n}\right)\left[\exp \left(i \Psi_{n}\right) z+\exp \left(-i \Psi_{n}\right) z^{*}\right]^{2} \\
& \times\left(e^{i\left(\omega-\psi_{N+1}\right)} z+e^{-i\left(\omega-\psi_{N+1}\right)} z^{*}\right) .
\end{aligned}
$$

The previous results can be combined to give

$$
\begin{aligned}
P_{N+1}= & e^{i \psi_{N+1}} P_{N}-\frac{i}{4} k_{N} e^{i \psi_{N+1}}\left\{\left[\exp \left(i \Psi_{N}\right) z+\exp \left(-i \Psi_{N}\right) z^{*}\right]^{2}+\sum_{n=0}^{N-1} k_{n} \sin \left(\omega-\psi_{N}-\Psi_{n}\right)\right. \\
& \left.\times\left[\exp \left(i \Psi_{n}\right) z+\exp \left(-i \Psi_{n}\right) z^{*}\right]^{2}\left(e^{i\left(\omega-\psi_{N+1}\right)} z+e^{-i\left(\omega-\psi_{N+1}\right)} z^{*}\right)\right\}
\end{aligned}
$$

so that 


$$
\begin{aligned}
P_{N+1}= & e^{i \omega}\left\{z-\frac{i}{4} \sum_{n=0}^{N} k_{n} \exp \left(-i \Psi_{n}\right)\left[\exp \left(i \Psi_{n}\right) z+\exp \left(-i \Psi_{n}\right) z^{*}\right]^{2}-\frac{i}{4} \sum_{m=0}^{N} k_{m} \sum_{j=0}^{m} k_{j} \exp \left(-i \Psi_{m}\right) \sin \left(\Psi_{m}-\Psi_{j}\right)\right. \\
& \left.\times\left[\exp \left(i \Psi_{m}\right) z+\exp \left(-i \Psi_{m}\right) z^{*}\right]\left[\exp \left(i \Psi_{j}\right) z+\exp \left(-i \Psi_{j}\right) z^{*}\right]^{2}\right\} .
\end{aligned}
$$

Therefore, $P_{N+1}$ is true and hence $P_{N}$ is true $\forall N$.

\section{APPENDIX C: ANALYTICAL COMPUTATION OF THE COMPOSITION OF A SET OF OCTUPOLAR MAPS}

In this Appendix the composition of $M$ maps representing the effect of octupole nonlinearities in the single-kick approximation [18] is described. As in Appendix B, normalized Courant-Snyder coordinates [15] are used and the 2D map from the $j$ th to the $(j+1)$ th octupolar element reads

$$
\left(\begin{array}{c}
x^{\prime} \\
p_{x}^{\prime}
\end{array}\right)=R\left(\psi_{j+1}\right)\left(\begin{array}{c}
x \\
p_{x}+k_{3, j} x^{3}
\end{array}\right) .
$$

In the following the notation $\chi_{n}=k_{3, n}$ will be used. The sequence of nonlinear magnetic elements can be represented as in Fig. 10. The element indicated with $k_{0}$ repre- sents a single sextupole, while the other elements are octupoles. Under these assumptions, the following result holds.

Theorem 2.-The composition of $M$ cubic maps of type (C1) in complex coordinates $\left(z, z^{*}\right)$ plus one single quadratic map of type (B1) is given, up to third order, by

$$
\begin{aligned}
z^{\prime}= & e^{i \omega}\left\{z-\frac{i}{4}\left(z+z^{*}\right)^{2}-\frac{i}{8} \sum_{n=0}^{N-1} \chi_{n} \exp \left(-i \Psi_{n}\right)\right. \\
& \left.\times\left[\exp \left(i \Psi_{n}\right) z+\exp \left(-i \Psi_{n}\right) z^{*}\right]^{3}\right\}
\end{aligned}
$$

Proof-CAs for the previous proposition, the theorem is proved by induction. The proposition $P_{1}$ is trivially shown to hold true. Then, one has to show that $P_{N}$ true implies $P_{N+1}$. It is clear that

$$
\begin{aligned}
P_{N+1}= & e^{i \psi_{N+1}}\left(z-\frac{i}{8} \chi_{N}\left(z+z^{*}\right)^{3}\right) \circ e^{i\left(\omega-\psi_{N+1}\right)}\left\{z-\frac{i}{4}\left(z+z^{*}\right)^{2}-\frac{i}{8} \sum_{n=0}^{N-1} \chi_{n} \exp \left(-i \Psi_{n}\right)\left[\exp \left(i \Psi_{n}\right) z+\exp \left(-i \Psi_{n}\right) z^{*}\right]^{3}\right\} \\
= & e^{i \omega}\left\{z-\frac{i}{4}\left(z+z^{*}\right)^{2}-\frac{i}{8} \sum_{n=0}^{N-1} \chi_{n} \exp \left(-i \Psi_{n}\right)\left[\exp \left(i \Psi_{n}\right) z+\exp \left(-i \Psi_{n}\right) z^{*}\right]^{3}\right\} \\
& -\frac{i}{8} \chi_{N} e^{i \psi_{N+1}\left[e^{i(\omega-\psi N+1)} z+e^{-i(\omega-\psi N+1)} z^{*}\right]^{3}} \\
= & e^{i \omega}\left\{z-\frac{i}{4}\left(z+z^{*}\right)^{2}-\frac{i}{8} \sum_{n=0}^{N-1} \chi_{n} \exp \left(-i \Psi_{i}\right)\left[\exp \left(i \Psi_{n}\right) z+\exp \left(-i \Psi_{n}\right) z^{*}\right]^{3}\right. \\
& \left.-\frac{i}{8} \chi_{N} e^{i\left(-\omega+\psi_{N+1}\right)}\left[e^{i\left(\omega-\psi_{N+1}\right)} z+e^{-i\left(\omega-\psi_{N+1}\right)} z^{*}\right]^{3}\right\} .
\end{aligned}
$$

The substitution $\omega=\sum_{i=0}^{N+1} \psi_{i}=\psi_{N+1}+\sum_{i=0}^{N} \psi_{i}$ gives the final result:

$$
P_{N+1}=e^{i \omega}\left\{z-\frac{i}{4}\left(z+z^{*}\right)^{2}-\frac{i}{8} \sum_{n=0}^{N} \chi_{n} \exp \left(-i \Psi_{n}\right)\left[\exp \left(i \Psi_{n}\right) z+\exp \left(-i \Psi_{n}\right) z^{*}\right]^{3}\right\} .
$$

Therefore, $P_{N+1}$ is true and, hence, $P_{N}$ is true $\forall N$.

\section{APPENDIX D: ANALYTICAL COMPUTATION OF $\Omega_{2}$}

In this Appendix the computation of the first nonresonant term is carried out based on the results of the previous Appendices. First, the situation assumed in Appendix C will be considered. It can be observed that the octupolar nonlinearities do not contribute to the second order part of $F\left(z, z^{*}\right)$, which is determined by the single quadratic map.
The coefficients can be found in Ref. [18], namely,

$$
\begin{aligned}
f_{2,0} & =-\frac{i}{4} e^{i \omega} \quad f_{1,1}=-\frac{i}{2} e^{i \omega} \quad f_{0,2}=-\frac{i}{4} e^{i \omega}, \\
\phi_{2,0} & =\frac{1}{8}\left[-\cot \left(\frac{\omega}{2}\right)+i\right] \quad \phi_{1,1}=\frac{1}{4}\left[\cot \left(\frac{\omega}{2}\right)+i\right] \\
\phi_{0,2} & =\frac{1}{8}\left[\cot \left(\frac{3 \omega}{2}\right)+i\right] .
\end{aligned}
$$

The only relevant third-order contribution is 


$$
f_{2,1}=-\frac{3}{8} i e^{i \omega} \sum_{n=0}^{N-1} \chi_{n}
$$

It is worthwhile pointing out that the term $f_{2,1}$ does not depend on the phase advance between the octupoles. Therefore, by direct application of Eqs. (A20) and (C2) one finds

$$
u_{2,1}=-\frac{i}{16} e^{i \omega}\left[3 \cot \left(\frac{\omega}{2}\right)+\cot \left(\frac{3 \omega}{2}\right)+6 \sum_{n=0}^{N-1} \chi_{n}\right]
$$

and then

$$
\Omega_{2}=-\frac{1}{16}\left[3 \cot \left(\frac{\omega}{2}\right)+\cot \left(\frac{3 \omega}{2}\right)+6 \sum_{n=0}^{N-1} \chi_{n}\right]
$$

In the case of the fourth-order resonance, $\omega=\pi / 2$, the condition $\Omega_{2}=0$ reads

$$
\sum_{n=0}^{N-1} \chi_{n}=-1 / 3
$$

The case of an array of sextupoles is more involved as the contributions to the second order of $F\left(z, z^{*}\right)$ have complex expressions which depend on the phase advance between elements. Using the following notation,

$$
\Theta_{+j}=\sum_{n=0}^{N-1} k_{n} e^{i j \Psi_{n}}=\Theta_{-j}^{*}
$$

and by direct application of Eq. (A5) and the result of Appendix B, the following holds:

$$
\begin{gathered}
f_{2,0}=-\frac{i}{4} e^{i \omega} \Theta_{+} \quad f_{1,1}=-\frac{i}{2} e^{i \omega} \Theta_{-} \\
f_{0,2}=-\frac{i}{4} e^{i \omega} \Theta_{-3} \quad \text { and } \quad \phi_{2,0}=\frac{f_{2,0}}{e^{2 i \omega}-e^{i \omega}} \\
\phi_{1,1}=\frac{f_{1,1}}{1-e^{i \omega}} \quad \phi_{0,2}=\frac{f_{0,2}}{e^{-2 i \omega}-e^{i \omega}} .
\end{gathered}
$$

Straightforward application of the functional equation (A5) gives, after some lengthy algebra,

$$
\begin{aligned}
f_{2,1}= & -\frac{i}{4} e^{i \omega} \sum_{m=0}^{N-1} k_{m} \sum_{j=0}^{m} k_{j} \exp \left(-i \Psi_{m}\right) \sin \left(\Psi_{m}-\Psi_{j}\right) \\
& \times\left[\exp \left(2 i \Psi_{j}\right) \exp \left(-i \Psi_{m}\right)+2 \exp \left(i \Psi_{m}\right)\right] \\
= & -\frac{i}{4} e^{i \omega} \sum_{m=0}^{N-1} k_{m} \sum_{j=0}^{m} k_{j} \sin \left(\Psi_{m}-\Psi_{j}\right) \\
& \times\left\{\exp \left[2 i\left(\Psi_{j}-\Psi_{m}\right)\right]+2\right\} .
\end{aligned}
$$

By direct substitution into Eq. (A20) one obtains

$$
\begin{aligned}
u_{2,1}= & -\frac{e^{i \omega}}{8}\left[-8 e^{-i \omega} f_{2,1}+\Theta_{+} \Theta_{-}\left(\frac{3 i}{2} \cot \frac{\omega}{2}-\frac{1}{2}\right)\right. \\
& \left.+\Theta_{+3} \Theta_{-3}\left(\frac{i}{2} \cot \frac{3 \omega}{2}+\frac{1}{2}\right)\right],
\end{aligned}
$$

where the relation $\left(e^{i k \omega}-1\right)^{-1}=-1 / 2-i / 2 \cot (k \omega / 2)$ was used. The final results reads

$$
\begin{aligned}
u_{2,1}= & -\frac{i}{8} e^{i \omega}\left\{\sum _ { m = 0 } ^ { N - 1 } k _ { m } \sum _ { j = 0 } ^ { m } k _ { j } \left\{3 \sin \left(\Psi_{m}-\Psi_{j}\right)\right.\right. \\
& \left.+\sin \left[3\left(\Psi_{m}-\Psi_{j}\right)\right]\right\}+\Theta_{+} \Theta_{-}\left(\frac{3}{2} \cot \frac{\omega}{2}\right) \\
& \left.+\Theta_{+3} \Theta_{-3}\left(\frac{1}{2} \cot \frac{3 \omega}{2}\right)\right\} .
\end{aligned}
$$

The use of the fundamental relation (A17) allows the determination of $\Omega_{2}$.

[1] R. Cappi and M. Giovannozzi, Phys. Rev. Lett. 88, 104801 (2002).

[2] R. Cappi and M. Giovannozzi, in Proceedings of the Eighth European Particle Accelerator Conference, edited by T. Garvey et al. (Institute of Physics, London, 2002), p. 1250 .

[3] R. Cappi and M. Giovannozzi, in Proceedings of the 2003 Particle Accelerator Conference, edited by J. Chew et al. (IEEE, Piscataway, NJ, 2003), p. 2910.

[4] R. Cappi and M. Giovannozzi, Phys. Rev. ST Accel. Beams 7, 024001 (2004).

[5] C. Bovet, D. Fiander, L. Henny, A. Krusche, and G. Plass, in Proceedings 1973 PAC, edited by D. W. Dupen (IEEE, New York, 1973), p. 438.

[6] R. Cappi and M. Giovannozzi, CERN Divisional Report No. CERN/PS 2002-083 (AE), 2002.

[7] J. Buon, in Proceedings of the CERN Accelerator School (CERN 94-01 Vol. 1, p. 89, 1994).

[8] R. Cappi, M. Giovannozzi, M. Martini, E. Métral, G. Métral, A.-S. Müller, and R. Steerenberg, in Proceedings of the 2003 Particle Accelerator Conference (Ref. [3]), p. 388 .

[9] M. Giovannozzi, R. Cappi, S. Gilardoni, M. Martini, E. Métral, A.-S. Müller, A. Sakumi, and R. Steerenberg, in Proceedings of the Ninth European Particle Accelerator Conference, edited by C. Petit-Jean-Genaz (Institute of Physics, London, 2004), p. 173.

[10] M. Giovannozzi, R. Cappi, S. Gilardoni, M. Martini, E. Métral, A.-S. Müller, P. Scaramuzzi, and R. Steerenberg, in 33rd ICFA Advanced Beam Dynamics Workshop on High Intensity and High Brightness Hadron Beams, AIP Conference Proceedings 773, edited by I. Hofmann, J.-M. Lagniel, and R.W. Hasse (AIP, Melville, NY, 2005), p. 296.

[11] R. Cappi, S. Gilardoni, M. Giovannozzi, M. Martini, E. Métral, A.-S. Müller, and R. Steerenberg, in Proceedings of the 2005 Particle Accelerator Conference, edited by C. Horak (IEEE, Piscataway, NJ, 2005), p. 117. 
[12] S. Gilardoni, M. Giovannozzi, M. Martini, E. Métral, A.S. Müller, P. Scaramuzzi, and R. Steerenberg, Phys. Rev. ST Accel. Beams 9, 104001 (2006).

[13] M. J. Barnes, O.E. Berrig, A. Beuret, J. Borburgh, P. Bourquin, R. Brown, J.-P. Burnet, F. Caspers, J.-M. Cravero, T. Dobers, T. Fowler, S. Gilardoni, M. Hourican, W. Kalbreier, T. Kroyer, F. di Maio, M. Martini, V. Mertens, E. Métral, K. D. Metzmacher, C. Rossi, J.-P. Royer, L. Sermeus, R. Steerenberg, G. Villiger, and T. Zickler, CERN Report No. 2006-011, 2006, edited by M. Giovannozzi.

[14] M. Giovannozzi and J. Morel, Phys. Rev. ST Accel. Beams 10, 034001 (2007).

[15] E. Courant and H. Snyder, Ann. Phys. (Paris) 3, 1 (1958).

[16] A. W. Chao, D. Johnson, S. Peggs, J. Peterson, C. Saltmarsh, L. Schachinger, R. Meller, R. Siemann, R. Talman, P. Morton, D. Edwards, D. Finley, R. Gerig, N. Gelfand, M. Harrison, R. Johnson, N. Merminga, and M. Syphers, Phys. Rev. Lett. 61, 2752 (1988).

[17] S. Y. Lee, M. Ball, B. Brabson, D. D. Caussyn, J. Collins, S. Curtis, V. Derenchuck, D. DuPlantis, G. East, M. Ellison, T. Ellison, D. Friesel, B. Hamilton, W. P. Jones, W. Lamble, D. Li, M. G. Minty, P. Schwandt, T. Sloan, G. Xu, A. W. Chao, S. Tepikian, and K. Y.Ng, Phys. Rev. Lett. 67, 3768 (1991).

[18] A. Bazzani, G. Servizi, E. Todesco, and G. Turchetti, CERN Yellow Report, 94-02, 1994.

[19] H. Poincaré, Les métodes nouvelles de la méchanique céleste (Gauthier-Villars, Paris, 1892).
[20] G. D. Birkhoff, Acta Math. 43, 1 (1922).

[21] J. Moser, Nachr. Akad. Wiss. Göttingen. Math.-Phys. Kl. II, 1 (1962).

[22] A. Dragt, Nucl. Instrum. Methods Phys. Res., Sect. A 258, 339 (1987).

[23] A. Bazzani, G. Turchetti, P. Mazzanti, and G. Servizi, Nuovo Cimento B 102, 51 (1988).

[24] E. Forest, M. Berz, and J. Irwin, Part. Accel. 24, 91 (1989).

[25] E. Forest, Beam Dynamics: A New Attitude and Framework (Harwood, Sidney, 1998).

[26] D. Quatraro, Master thesis, University of Bologna, 2007.

[27] W. E. Gabella, J. Rosenzweig, R. Kick, and S. Peggs, in Proceedings of the 1993 Particle Accelerator Conference, edited by C. W. Leemann et al. (IEEE, Piscataway, NJ, 1993), p. 233.

[28] E. Regenstreif, CERN Yellow Report No. 58-6, 1958.

[29] J.-P. Burnet, M. Giovannozzi, E. Métral, O. Michels, R. Steerenberg, and B. Vandorpe, in Proceedings of the Tenth European Particle Accelerator Conference, edited by C. Petit-Jean-Genaz (Institute of Physics, London, 2006), p. 264.

[30] R. Cappi, M. Giovannozzi, M. Martini, E. Métral, G. Métral, A.-S. Müller, and R. Steerenberg, in Proceedings of the 2003 Particle Accelerator Conference (Ref. [3]), p. 2913.

[31] C. K. Allen and T. P. Wangler, Phys. Rev. ST Accel. Beams 5, 124202 (2002). 